\title{
Intrachain photoluminescence properties of conjugated polymers as revealed by long oligothiophenes and polythiophenes diluted in an inactive solid matrix
}

Katsuichi Kanemoto, Tatsuji Sudo, Ichiro Akai, Hideki Hashimoto, Tsutomu Karasawa, Yoshio Aso, Tetsuo Otsubo

\begin{tabular}{|c|l|}
\hline Citation & Physical Review B, 73(23); 235203 \\
\hline Issue Date & 2006-06-15 \\
\hline Type & Journal Article \\
\hline Textversion & Publisher \\
\hline Rights & $\begin{array}{l}\text { CAmerican Physical Society. This article may be downloaded for personal use } \\
\text { only. Any other use requires prior permission of the author and American Physical } \\
\text { Society. } \\
\text { The following article appeared in Physical Review B Vol.73, Iss.23 and may be } \\
\text { found at https://doi.org/10.1103/PhysRevB.73.235203 }\end{array}$ \\
\hline DOI & \begin{tabular}{l}
$10.1103 /$ PhysRevB.73.235203 \\
\hline
\end{tabular} \\
\hline
\end{tabular}

Self-Archiving by Author(s) Placed on: Osaka City University

KANEMOTO, K., SUDO, T., AKAI, I., HASHIMOTO, H., KARASAWA, T., ASO, Y., \& OTSUBO, T. (2006). Intrachain photoluminescence properties of conjugated polymers as revealed by long oligothiophenes and polythiophenes diluted in an inactive solid matrix. Physical Review B. 73, 235203. https://doi.org/10.1103/PhysRevB.73.235203 


\title{
Intrachain photoluminescence properties of conjugated polymers as revealed by long oligothiophenes and polythiophenes diluted in an inactive solid matrix
}

\author{
Katsuichi Kanemoto, ${ }^{1, *}$ Tatsuji Sudo, ${ }^{1}$ Ichiro Akai, ${ }^{1}$ Hideki Hashimoto, ${ }^{1,2}$ Tsutomu Karasawa, ${ }^{1}$ Yoshio Aso, ${ }^{3}$ and \\ Tetsuo Otsubo 4 \\ ${ }^{1}$ Department of Physics, Graduate Schol of Science, Osaka City University, 3-3-138 Sugimoto, Sumiyoshi-ku, Osaka 558-8585, Japan \\ ${ }^{2}$ PRESTO, Japan Science and Technology Agency, 4-1-8 Honcho Kawaguchi, Saitama 332-0012, Japan \\ ${ }^{3}$ The Institute of Scientific and Industrial Research, Osaka University, Ibaraki, Osaka 567-0047, Japan \\ ${ }^{4}$ Department of Applied Chemistry, Graduate School of Engineering, Hiroshima University, Higashi-hiroshima, 739-8527, Japan
}

(Received 24 November 2005; revised manuscript received 21 March 2006; published 6 June 2006)

\begin{abstract}
The intrinsic intrachain photoluminescence (PL) dynamics of conjugated polymers in the solid state is investigated. We focus on the PL properties of long $\beta$-substituted oligothiophenes (8-mer, 12-mer, and 16-mer) and regio-regular (RR) and regio-random poly(3-octylthiophenes) (P3OTs) diluted in the inactive solid matrix polypropylene (PP). The oligothiophenes have well-resolved 0-0 PL and absorption peaks at $4 \mathrm{~K}$ and show a good linear relationship with the reciprocal of the ring number. Franck-Condon analyses on the PL spectra reveal that the strength of the electron-phonon coupling represented by the Huang-Rhys factor becomes weak with increasing chain length in the oligomers. In contrast, the presence of stronger electron-phonon coupling is confirmed in RR P3OT despite it being of a much longer chain length than oligomers. This is probably due to its stereoregular chain conformation. The materials used in this work diluted in PP exhibit single-exponential PL decays with lifetimes that decrease with increases in the chain length. The presence of intrachain exciton migration is shown by the time-resolved PL measurements, except for the case of RR P3OT. The energy shifts attributed to intrachain migration are much smaller than those ascribed to interchain migration. We conclude that a change in the torsion angle around the thiophene ring bonds occurs within $50 \mathrm{ps}$ following the absorption transition in thiophene derivatives and that the torsional change gives rise to a constant Stokes shift (60-70 meV), regardless of the chain length. We propose that the Stokes shift can be utilized for estimating the magnitude of exciton migration in thiophene derivatives, even in the case of undiluted films.
\end{abstract}

DOI: 10.1103/PhysRevB.73.235203

PACS number(s): 73.61.Ph, 78.47.+p, 78.55.Kz

\section{INTRODUCTION}

$\pi$-conjugated polymers have been the subject of much interest due to their potential optoelectronic applications such as light-emitting diodes (LEDs), solar cells, and fieldeffect transistors. ${ }^{1-3}$ As a result, the electronic states of $\pi$-conjugated polymers have been studied using a variety of spectroscopic techniques. ${ }^{4-6}$ However, accurate spectral characterization of these conjugated polymer systems is difficult to obtain due to their complicated electronic states that originate from a number of uncertain structural parameters: partial interchain interactions, inhomogeneous chain length, and chain distortion. In order to better understand the electronic states in conjugated polymers, it is therefore desirable to study different systems using multiple experimental approaches.

Polymer chains in conjugated polymers are usually distributed amorphously in the solid film. The resulting interchain interactions play an essential role in charge transport in semiconductor devices. Due to the presence of these interchain interactions, the electronic states become delocalized over several molecules, making it difficult to characterize them. Potentially, one of the most effective ways to explore the electronic state in a conjugated polymer would be first to investigate the fundamental properties of an isolated polymer. Then the electronic state would be probed as a function of increasing interchain interactions. In order to characterize the physical properties of an isolated polymer many works have been carried in solution. ${ }^{7-10}$ In this present work, we particularly focus on a system where an inactive solid matrix is used for dilution because it can be regarded as a solid dilution model of neat (undiluted) films.

The use of solid matrices for dilution purposes have been reported for conjugated polymers. ${ }^{11-17}$ In these studies transparent polymers were preferred as the solid matrix. However, it should be noted that polymer blends can easily result in phase separation due to low mixing entropy and hence careful judgment is required to ensure a homogenous polymer blend. Indeed, we recently reported that head-to-tail coupled regio-regular (RR) poly(3-octylthiophene) (P3OT), known to have a self-assembling property, ${ }^{7,18}$ leads to phase separation due to imperfect miscibility when blended with the widely used transparent polymer poly-(methyl methacrylate) (PMMA). ${ }^{17}$ Furthermore, when compared to its neat spincoated film, it has been demonstrated that when P3OT is blended with saturated hydrocarbon polymers such as polyethylene (PE) or polypropylene (PP), it gives rise to a blueshifted absorption spectra. This indicates that the hydrocarbon polymers are likely to be effective in the formation of a homogeneous polymer blend with the conjugated polymer with alkyl substituents. ${ }^{16,17}$ Also, such a saturated hydrocarbon polymer is an ideal inactive support matrix for a conjugated polymer because its hydrocarbons are expected to have negligible interaction with the $\pi$-electron backbone in conjugated molecule.

In order to draw the fundamental properties of a conjugated polymer, the use of materials with defined chain lengths, i.e., oligomers, is desirable as they are expected to 
reduce the effect of chain-length inhomogeneity, which often veils the optical properties of the polymer. Conjugated oligomers have been widely studied because of their suitability as models for their related polymers as well as for their potential use in the nanoscale industry. The oligothiophenes are a well-studied series of conjugated oligomers. ${ }^{19}$ Actually, nonsubstituted, $\alpha$-linked oligothiophenes (up to the 7-mer) have often been used for investigating the chain-length dependence on the optical properties of polymers, including the position of the transition energy. ${ }^{20-24}$ However, when an oligomer is used to model its polymer homologue, it raises the question whether the chain length is sufficiently long enough in order to accurately model the electronic state of the polymer. Related to this, the incorporation of soluble alkyl substituent groups at the $\beta$ position was shown to be effective in elongating the oligomer length and recently led to the development of long (up to the 48-mer) $\beta$-substituted oligothiophenes. ${ }^{25-27}$ These studies showed that due to enhanced conjugation length the absorption peak energy of the $\beta$-substituted oligothiophenes in solution decreases with increasing the chain length. Moreover, the displacement of the peak energy becomes minor for oligomers greater than $\sim 10$-mer. These results indicate that oligomers that are larger than $\sim 10$-mer can be used as suitable models for studying their conjugated polymer counterparts.

In order to clarify the fundamental photoluminescence (PL) process in conjugated polymers, we address "intrachain" PL dynamics of conjugated polymers and oligomers based on the analyses of steady-state and time-resolved PL spectra of long oligothiophenes, as well as polythiophene (PT) derivatives. In this work, we adopted the use of PP as an inactive matrix as it is effective to form a homogeneous polymer blend with PT. Furthermore, a series of long oligothiophenes (8-, 12-, and 16-mers) have been studied since they are considered to be suitable models for investigating polymer characteristics and can highlight the dependence of chain length on the overall optical properties of the system.

So far the PL dynamics of conjugated polymers has been studied on the basis of results obtained in neat films of poly $\left(p\right.$-phenylenevinylene) $\quad(\mathrm{PPV}),{ }^{28,29}$ polyfluorene (PFO), ${ }^{30,31}$ and PT derivatives. ${ }^{32,33}$ Two remarkable features were derived from these time-resolved PL measurements: (1) a nonexponential PL decay and (2) the exciton migration effect. In the measurements where PT derivatives were used, the above features were interpreted as resulting from intrachain processes. ${ }^{32,33}$ In this present work we show that isolated PT derivatives fail to exhibit nonexponential PL decays and exciton migration effects. This clearly indicates that these phenomena cannot be ascribed to intrachain events. Furthermore, we characterize the intrinsic intrachain PL properties in conjugated polymers based on the analyses of time-resolved and steady-state PL spectra for a variety of thiophene samples, which is expected to present further insights for an understanding of the PL mechanism in "neat" samples. Finally, we show that strong electron-phonon coupling and stereoregularity in the chain conformation are important factors to determine the intrachain electronic states in conjugated polymer.

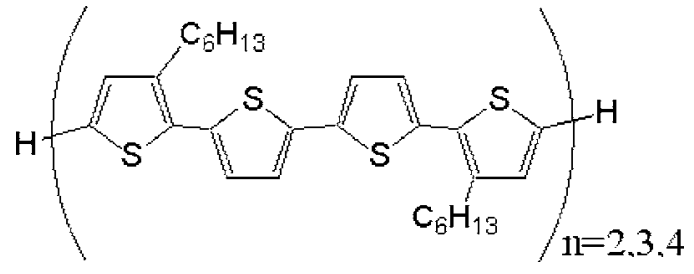

FIG. 1. The chemical structure of oligothiophenes used in this study: $n=2$ for the 8 -mer $(8 \mathrm{~T}), n=3$ for the 12 -mer $(12 \mathrm{~T})$, and $n=4$ for the 16 -mer $(16 \mathrm{~T})$, where $n$ is the number of repetitions.

\section{EXPERIMENTAL}

We used two types of polythiophenes, RR and regiorandom (RRa) P3OTs, that were purchased from Aldrich. The synthesis of these polymers as well as their fundamental properties have been reported elsewhere. ${ }^{7}$ The oligomer samples used in this study were the 8-mer, 12-mer-, and 16mer, $\alpha$-linked oligothiophenes (hereafter abbreviated as $8 \mathrm{~T}$, $12 \mathrm{~T}$, and $16 \mathrm{~T}$, respectively) with two hexyl-substituents at the $\beta$ positions per four thiophene rings (Fig. 1). The synthesis of these oligomers has been reported previously. ${ }^{34}$ Dilution of P3OTs, or oligothiophenes, was carried out using amorphous PP (Aldrich) by dissolving them in xylene with a guest/PP weight ratio of $1 / 30000$ as described previously. ${ }^{17}$

Absorption spectra were recorded with a Shimadzu UV2400 spectrophotometer. The samples were semitransparent in the region of the absorption measurements due to scattering by the PP particles. Control spectra of PP were measured and subtracted from the absorption spectra of the guest-PP samples. Although the background was not entirely removed it was sufficient to permit the identification of the absorption peaks attributed to the different oligomers used in this study. Continuous wave (CW) PL measurements were performed using an $\mathrm{Ar}^{+}$laser as a light source at $488 \mathrm{~nm}(\sim 10 \mathrm{~mW})$. PL excitation (PLE) measurements were performed using a Xe lamp as a light source attached to a monochromator with a double grating (Spex, 270). PL and PLE spectra were corrected for wavelength sensitivity by comparison with a standard tungsten lamp calibrated with NBS (No. EPT-1285). The samples used in this work had optical densities of $\sim 0.1$ and hence self-absorption was assumed to have a negligible influence on the experimental data.

In the time-resolved PL experiments, samples were excited with the second harmonic generation (SHG) output $(400 \mathrm{~nm})$ from a Ti:sapphire regenerative amplifier system (Spectra Physics, Hurricane-X) supplying 100-fs pulses at $800 \mathrm{~nm}$ with a repetition rate of $1 \mathrm{kHz}$. Samples were irradiated at the fluence giving a linear PL response. Timeresolved signals were detected with a streak camera (Hamamatsu, C2909) triggered by a photodiode that enabled us to record a $90-\mathrm{nm}$ range of time-resolved signals by combining it with a monochromator. The time resolution of the system was approximately $20 \mathrm{ps}$. Decay profiles were recorded by integrating the time-resolved signals over the 90-nm range.

\section{RESULTS}

\section{A. Absorption and steady-state PL spectra}

Figure 2 shows the absorption, PL, and PLE spectra for the RR and RRa P3OTs in PP measured at $4 \mathrm{~K}$. Both P3OTs 


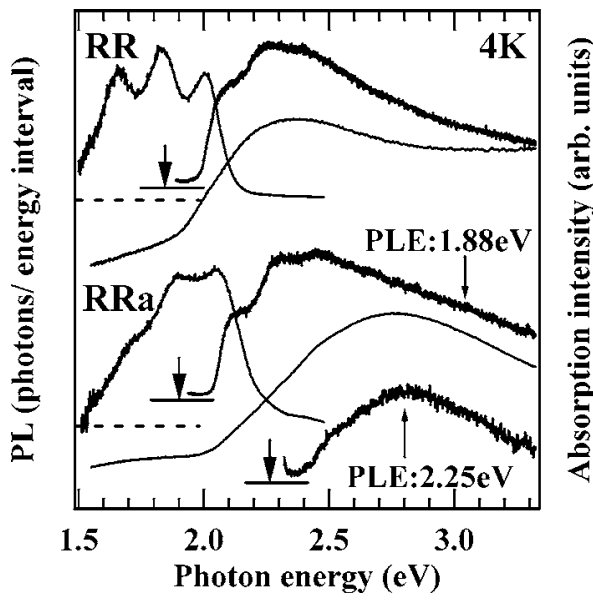

FIG. 2. The absorption (lower right), photoluminescence excitation (PLE) (upper right), and steady-state PL (left) spectra excited at $2.54 \mathrm{eV}$ at $4 \mathrm{~K}$ for regio-regular (RR) and regio-random (RRa) poly(3-octylthiophene)s (P3OTs) diluted in polypropylene (PP). The horizontal solid and dotted lines represent the level of the baselines for the PLE and PL spectra, respectively. The arrows indicate the energy positions at which the PLE spectra were measured. The PLE spectrum for RR was measured at the second vibronic peak $(1.82 \mathrm{eV})$. The PLE spectra for RRa were measured at the second vibronic peak $(1.88 \mathrm{eV})$ and at $2.25 \mathrm{eV}$.

exhibit well-resolved PL peaks at low temperature and have more defined spectral structures than previous measurements carried out at room temperature. ${ }^{17}$ The PL peaks were always observed as the major ones, even under excitation at $400 \mathrm{~nm}$. The highest energy peak for each P3OT is assigned to the $0-0$ peak and their values determined; see Table. I. The absorption spectrum of each P3OT gives only a single broad band but resolved peaks are obtained from their PLE spectra. At this point it should be noted that the absorption spectrum of RRa and its PLE spectrum when measured at the second vibronic peak $(1.88 \mathrm{eV})$ do not have similar peak characteristics but they are similar when the PLE spectrum is measured at $2.25 \mathrm{eV}$. This indicates that two, or more, species with different effective conjugation lengths (ECLs) are present in the RRa-P3OT sample. Hence, the PLE spectrum at $1.88 \mathrm{eV}$ is a substitute for an absorption spectrum of species with longer ECLs that give rise to the primary PL. The PL shoulder at approximately $2.25 \mathrm{eV}$ originates from species with shorter ECLs that have an absorption peak at $2.8 \mathrm{eV}$. Detailed analyses on the PL dynamics, including results of time-resolved PL, are described in the Sec. IV B.

TABLE I. The observed 0-0 peaks of absorption and PL spectra and their Stokes shifts measured at $4 \mathrm{~K}$.

\begin{tabular}{cccccc}
\hline \hline Sample & $8 \mathrm{~T}$ & $12 \mathrm{~T}$ & $16 \mathrm{~T}$ & $\mathrm{RR}$ & $\mathrm{RRa}$ \\
\hline $\begin{array}{c}\text { Absorption } \\
(\mathrm{eV})\end{array}$ & 2.32 & 2.21 & 2.16 & $\sim 2.08^{\mathrm{a}}$ & $\sim 2.13^{\mathrm{a}}$ \\
PL (eV) & 2.26 & 2.14 & 2.10 & 2.01 & 2.07 \\
$\begin{array}{c}\text { Stokes shift } \\
(\mathrm{eV})\end{array}$ & 0.06 & 0.07 & 0.06 & $\sim 0.07$ & $\sim 0.06$ \\
\hline \hline
\end{tabular}

a Determined from the PL-excitation spectrum.

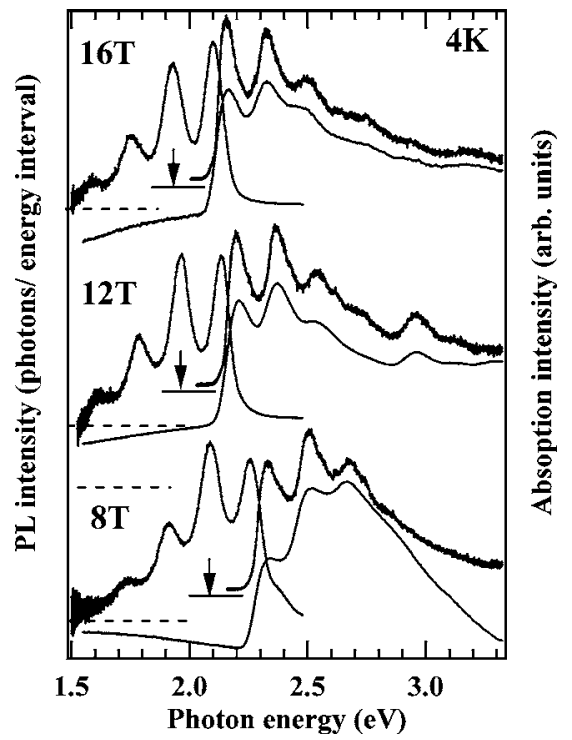

FIG. 3. The absorption (lower right), photoluminescence excitation (PLE) (upper right), and steady-state PL (left) spectra excited at $2.54 \mathrm{eV}$ at $4 \mathrm{~K}$ for oligothiophenes $(8 \mathrm{~T}, 12 \mathrm{~T}$, and $16 \mathrm{~T})$ diluted in PP. The horizontal solid and dotted lines represent the level of the baselines for the PLE and PL spectra, respectively. The arrows indicate the energy positions at which the PLE spectra were measured. The PLE spectra were measured at the second vibronic peak; $2.09 \mathrm{eV}$ for $8 \mathrm{~T}, 1.97 \mathrm{eV}$ for $12 \mathrm{~T}$, and $1.93 \mathrm{eV}$ for $16 \mathrm{~T}$.

In the RR sample diluted in PP, the PLE spectrum at the second PL peak $(1.82 \mathrm{eV})$ is observed near to the absorption spectrum and there is no apparent indication of the presence of additional species. Furthermore, both the PLE and PL peaks are well defined with the lowest and highest energy peaks assigned to the $0-0$ peaks of the absorption and PL spectra, respectively. Recently, the absorption and PLE peaks of RR diluted in PP were reported to have a redshift to the absorption peak in solution at room temperature. ${ }^{17}$ It was suggested that this was due to a rodlike chain morphology of RR in PP. ${ }^{17}$ Thus, the observed $0-0$ peaks in the RR sample (Fig. 2) are interpreted as originating from species existing in chains with a rodlike morphology. Shown in Fig. 3 are the absorption, PL, and PLE spectra of $8 \mathrm{~T}, 12 \mathrm{~T}$, and $16 \mathrm{~T}$ in $\mathrm{PP}$ measured at $4 \mathrm{~K}$. It should be noted that in all the oligothiophene samples the absorption, as well as the PL spectra, exhibit well-resolved peaks. The PLE spectra, recorded at the second PL peak, also show resolved peaks whose energies correspond to those of the structures found in their related absorption spectra; see Table I. The peaks in the oligothiophene samples with a constant spacing of $\sim 0.17 \mathrm{eV}$ are ascribed to vibronic splitting. To our knowledge, absorption spectra with well-resolved 0-0 peaks have never been previously reported for polythiophenes, or long oligothiophenes, in this dilution system.

Figure 4 displays the plots of the $0-0$ peaks of both absorption and PL spectra in the oligomer samples versus the reciprocal of ring number, $m$. Linear relations are confirmed in both the absorption and PL plots and the results of linear fits are given as below,

$$
E(\mathrm{eV})=2.00+2.57 / m \quad \text { (for absorption), }
$$




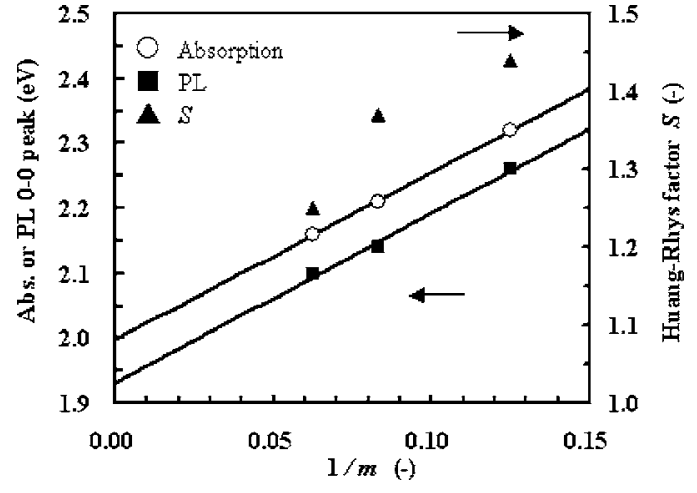

FIG. 4. A plot containing the 0-0 peaks of the absorption and PL spectra (left) and Huang-Rhys factors $S$ (right) at $4 \mathrm{~K}$ for the $8 \mathrm{~T}$, $12 \mathrm{~T}$, and $16 \mathrm{~T}$ versus the reciprocal of ring number $m$. The straight lines represent the linear fits of the $0-0$ peak data set.

$$
E(\mathrm{eV})=1.93+2.60 / m \quad \text { (for PL) } .
$$

Here, as has been proposed previously, ${ }^{35}$ the slope of the fit reflects the degree of coplanarity in the chain. Hence this value is expected to increase with the resonance integral between adjacent rings. The similarity of the values of the two slopes suggests that the coplanarity of the thiophene rings is almost maintained in the absorption and PL transitions. Furthermore, the results of the PL measurement provides conclusive evidence that the photoluminescence in this system occurs from the excited states whose electron distribution is directly correlated with extended chain length, i.e., PL does not occur from localized areas such as defects or trapped sites.

The left-hand $y$-axis intercept of the linear fits represents the $0-0$ peak energy of the oligomer with infinite ring number. However, the intercept values determined here are somewhat smaller than the 0-0 peak energies of the RR- and RRaP3OT samples shown in Table I. This finding can be attributed to the shrinkage of the ECL in the polymer samples, or to the difference in the substituent position among the samples, as has already been shown with a systematic study on PT derivatives. ${ }^{36}$ In contrast, if we adopt the absorption-peak energy dependence of $E(\mathrm{eV})=2.54$ $+3.15 / \mathrm{m}$ in head-to-tail octyl-substituted oligothiophenes in solution $^{35}$ and then subtract the energy of $\sim 0.5 \mathrm{eV}$ from $E(\mathrm{eV})$, the value which is defined as absorption-peak energy difference in the oligomer sample between the 0-0 peak energy at $4 \mathrm{~K}$ and the peak energy in solution [e.g., $2.82 \mathrm{eV}$ in $8 \mathrm{~T}$ (Ref. 34)], the intercept of linear fit for the 0-0 absorption peak of the head-to-tail oligothiophene is at $2.04 \mathrm{eV}$. This value is nearly equal to the $0-0$ absorption peak energy of RR P3OT in PP $(2.08 \mathrm{eV})$. We therefore emphasize that Eqs. (1) and (2) afford a good prediction of the 0-0 peak energy of absorption and PL spectra at $4 \mathrm{~K}$, respectively, in alkyl-substituted oligothiophenes and polythiophenes, when approximately $0.5 \mathrm{eV}$ energy is subtracted from the $0-0$ peak energy in solution.

\section{B. Time-resolved PL}

The PL time profiles of the $12 \mathrm{~T}, 16 \mathrm{~T}, \mathrm{RR}$, and RRa in $\mathrm{PP}$ were obtained from time-resolved PL measurements at

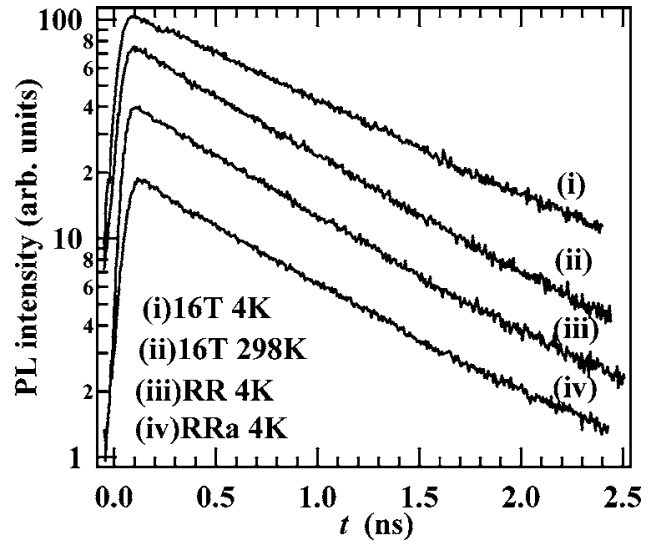

FIG. 5. The PL time profiles of (i) $16 \mathrm{~T}$ at $4 \mathrm{~K}$, (ii) $16 \mathrm{~T}$ at 298 K, (iii) RR-P3OT at 4 K, and (iv) RRa P3OT at 4 K, diluted in PP.

$4 \mathrm{~K}$ and at $298 \mathrm{~K}$. For clarity, Fig. 5 only displays the PL time profiles obtained from for RR P3OT (4 K), RRa P3OT $(4 \mathrm{~K})$, and $16 \mathrm{~T}(4 \mathrm{~K}$ and $298 \mathrm{~K})$. The linear response of the slopes indicates that all of the transient signals decay exponentially with a single lifetime. The derived values for all the samples are shown in Table II. These findings therefore indicate that conjugated polymers and long oligomers exhibit a single-exponential PL decay when appropriately diluted in a solid matrix, similarly to the case in liquid solution that has been reported for many polymer samples. In contrast, nonexponential-type PL decay was reported for neat solid films of conjugated polymers ${ }^{28-33}$ and such a decay process was also identified for neat solid films of the materials used in this study. ${ }^{37}$ Therefore we may conclude that the nonexponential PL decay is caused by an "interchain" process. It should be emphasized that in order to observe singleexponential PL decay kinetics at least a 1000-fold dilution (per weight) is necessary for the RR-P3OT/PP blend. ${ }^{16}$ The dilution factor should thus be chosen carefully in order to permit the observation of intrinsic intrachain PL processes. Indeed, the somewhat nonexponential PL decay reported previously for a $20 \%$ blend of a PPV-derivative/polycarbonate $(\mathrm{PC})^{38}$ may be attributed to the imperfect isolation of polymer chains in such a high sample concentration.

The data in Table II indicate several interesting features concerning the PL lifetime of the conjugated oligomers and polymers. Firstly, there is no significant difference in the lifetime between $12 \mathrm{~T}$ and $16 \mathrm{~T}$ and between RR and RRa P3OTs. The lifetimes of $12 \mathrm{~T}$ and $16 \mathrm{~T}$ are somewhat longer than those of the P3OTs at $4 \mathrm{~K}$. Furthermore, every sample listed in the table exhibits a decrease in the lifetime with increasing temperature, and the difference in the lifetime be-

TABLE II. The PL lifetimes/ps (and their inverse $/ 10^{9} \mathrm{~s}^{-1}$ ) at $4 \mathrm{~K}$ and $298 \mathrm{~K}$ for oligothiophenes $(8 \mathrm{~T}, 12 \mathrm{~T}$, and $16 \mathrm{~T})$ and $\mathrm{RR}$ P3OT and RRa P3OT diluted in PP.

\begin{tabular}{ccccc}
\hline \hline Sample & $12 \mathrm{~T}$ & $16 \mathrm{~T}$ & $\mathrm{RR}$ & $\mathrm{RRa}$ \\
\hline $4 \mathrm{~K} / \mathrm{ps}$ & $970(1.03)$ & $980(1.02)$ & $810(1.23)$ & $820(1.22)$ \\
$298 \mathrm{~K} / \mathrm{ps}$ & $790(1.27)$ & $790(1.27)$ & $730(1.37)$ & $760(1.32)$ \\
\hline \hline
\end{tabular}



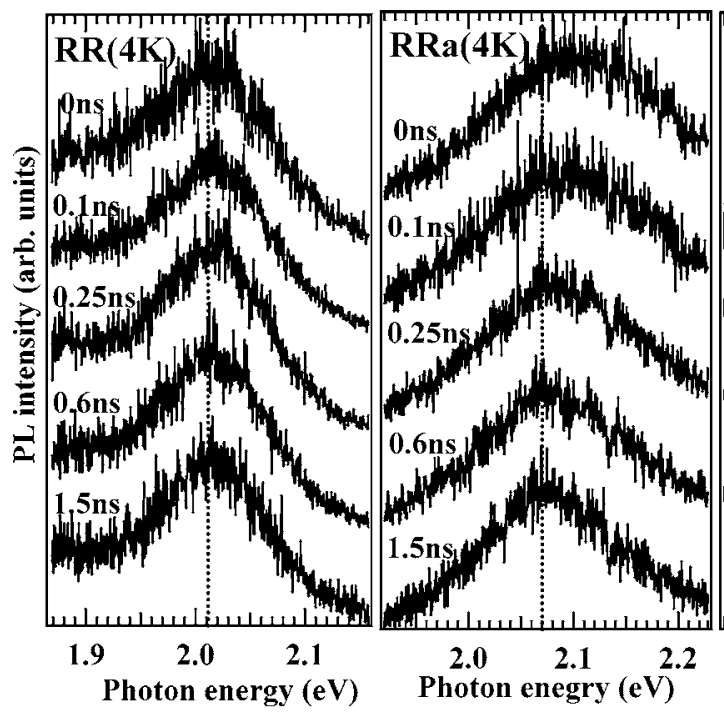

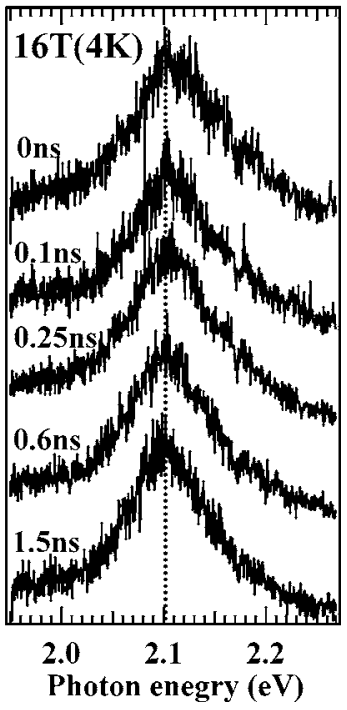

FIG. 6. The time-resolved PL spectra measured at $4 \mathrm{~K}$ for RR P3OT, RRa P3OT, and $16 \mathrm{~T}$ diluted in PP. The spectra were obtained by integrating signals over given time width. From the top, the integrated times are $0 \pm 50 \mathrm{ps}$, $100 \pm 20 \mathrm{ps}, \quad 250 \pm 40 \mathrm{ps}, \quad 600 \pm 50 \mathrm{ps}, \quad$ and $1500 \pm 100 \mathrm{ps}$. The average time in each spectrum is indicated in the figures. The spectra were scaled as to give nearly the same PL intensity. The dotted lines in the figures indicate the PL peak position in the steady-state PL spectrum. tween the oligomers and P3OTs reduces at $298 \mathrm{~K}$. In the case of single-exponential PL decay the process can simply be divided into two terms, $1 / \tau_{p l}=k_{r}+k_{n r}$, where $\tau_{p l}$ is the PL lifetime and $k_{r}$ and $k_{n r}$ are the radiative and the nonradiative decay rate constants, respectively. Here, the radiative rate constant of oligothiophenes in solution (up to the 7-mer) was reported to be almost independent of the oligomer length. ${ }^{23}$ Hence a variation in $k_{r}$ dependent on the chain length cannot be expected. Accordingly, we consider that the difference in the PL lifetime between our oligomer and P3OT samples is primarily attributed to the difference in the nonradiative decay constants $k_{n r}$.

Our observations can therefore be regarded as an increase of $k_{n r}$ concomitant with an increase of chain length. In contrast, the value of $k_{n r}$ of the shorter oligothiophenes was shown to decrease with increasing the chain length (up to the 4-mer) and then become almost constant for chain lengths greater than the 5 -mer. ${ }^{23}$ In addition, intersystem crossing (ISC) from the singlet $S_{1}$ to the triplet manifold $T_{i}$ was reported to make the main contribution to the nonradiative process when compared to other mechanisms containing an internal conversion (IC) event. ${ }^{23}$ It was suggested that in PT the ISC occurs efficiently when in solution. ${ }^{39}$ In our samples, the contribution of ISC could thus be dominant among possible nonradiative processes. In that case, the rate of ISC is primarily dependent on the energy difference between the $S_{1}$ and $T_{n}$ states ( $n$ is assigned to the nearest triplet state to $S_{1}$; say, $n=4$ for shorter oligomers ${ }^{40}$ ). However, when the $S_{1}$ and $T_{n}$ states give a similar chain-length dependence, their energy difference should be constant for an increment in the chain length. Therefore we conclude that a variation in the ISC process is not the primary origin of the increase in $k_{n r}$ with increasing chain length. Yet, if other ISC paths that do not lead to the nearest neighboring $T_{n}$ state are generated with increasing the chain length, the ISC process may increase $k_{n r}$ effectively with an increment of chain length.

In contrast, not only intrinsic events such as ISC, or IC, but extrinsic events could be included as the potential origin of the $k_{n r}$ increase with increasing chain length. In fact, it is possible for polymer chains in the solid state to have extrinsic or structural defects as well as morphological trapping sites. These defects and trapping sites can work as quenching sites of excitons and might be employed as a possible nonradiative pathway. Here, assuming that the number of quenching sites per chain length is constant and that the excitons can rapidly move within an individual chain to find a quenching site, the probability of the excitons to be quenched is expected to simply increase with increasing chain length. This may be true for the samples studied in this present work and hence we suggest that this mechanism is one of the origins of the observed $k_{n r}$ increase with chainlength increment.

In addition, the decreases at $298 \mathrm{~K}$ in $\tau_{p l}$ of the oligomers and P3OTs, resulting in close $\tau_{p l}$ values between them, indicate that nonradiative processes are enhanced as the temperature increases, when ignoring a temperature-dependent variation in $k_{r}$. With respect to the nonradiative processes mentioned above, if the ISC, or the extrinsic, processes have similar activation energies then both of them can be activated more or less by heat. It was previously reported that the ISC rate is not different between the PT derivative and the 12mer oligomer in solution at room temperature. ${ }^{41}$ When the reported rate constant of $k_{\mathrm{nr}}=1.2 \mathrm{~ns}$ is adopted for our samples, the radiative lifetimes are $2.3 \mathrm{~ns}$ for the oligomers and $2.0 \mathrm{~ns}$ for the P3OTs. These two values are close to the previously reported radiative lifetimes of shorter oligomers ${ }^{23}$ and of PT derivatives. ${ }^{36}$ Therefore, we conclude that the decreases in $\tau_{p l}$ at $298 \mathrm{~K}$ for the oligomers and P3OTs is primarily caused by the enhancement of the ISC process by heat. This indicates that ISC plays an important role in the nonradiative process at room temperature in both oligothiophene and polythiophene derivatives.

Figure 6 shows time-resolved PL spectra at $4 \mathrm{~K}$ for RR and RRa P3OTs and $16 \mathrm{~T}$ diluted in PP. Slight spectral redshifts with time are identified except for the case of RR P3OT. The spectral shifts can be displayed more quantitatively by shifts in the calculated spectral first moment of each spectrum; see Fig. 7. Figure 7 also shows a slight blueshift with time in RR P3OT. It is probably due to the contamination of a slight quantity of aggregate species normally giving redshifted and faster decaying PL signals. ${ }^{16}$

With relation to our present study, time-dependent spectral redshifts have been reported on the time-resolved PL 


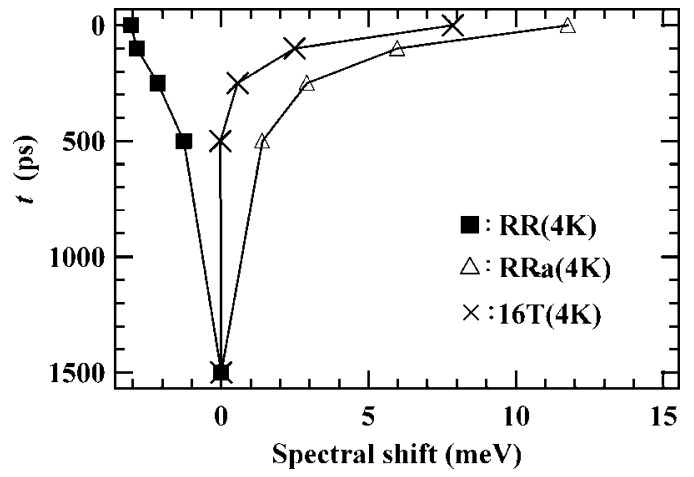

FIG. 7. Plots of the average spectral shift of the spectra shown in Fig. 6 based on the determination of the spectral first moment. The spectral shift is defined with a deviation from the moment in the spectrum at $1500 \pm 100$ ps that has a peak near to that of each steady-state PL spectrum.

spectra in slightly diluted PPV oligomers ${ }^{42}$ and in neat films of several conjugated polymers. ${ }^{28-33}$ The redshifts observed in our materials, however, are much smaller than the shifts previously reported. This difference is attributed to whether interchain or intrachain processes contribute to the observed spectral properties. The larger spectral shifts reported for the neat film samples are thus assigned to originating from interchain processes. Moreover, excitons generated in isolated chains should result only in a small spectral shift in timeresolved PL.

\section{DISCUSSION}

\section{A. Franck-Condon analyses for PL spectra}

The diluted samples that we used in this work are considered to have negligible interchain interactions between neighboring guest molecules. In this sense, we can regard that the one-dimensional (1D) electronic system is almost realized for our samples. One of the most remarkable features in the 1D electronic system is its strong electronphonon coupling, which has been actually taken into consideration for explaining a variety of optical experiments in conjugated polymers. ${ }^{43,44}$ The presence of the electronphonon couplings for our materials is demonstrated in Figs. 2 and 3 in the optical spectra that display a vibronic series. Here we discuss the extent of the electron-phonon coupling for our materials through a Franck-Condon (FC) analysis on their optical spectra.

The absorption and PL spectra with phonon replica $I(\omega)$ are generally expressed by the following formula: ${ }^{45,46}$

$$
I(\omega) \propto \sum_{n_{i}} \prod_{i} \frac{S_{i}^{n_{i}} \exp \left(-S_{i}\right)}{n_{i} !} \Gamma\left(\omega-\omega_{00} \pm \sum_{i} n_{i} \omega_{p, i}\right) .
$$

Here $S_{i}$ is the Huang-Rhys (HR) factor, $\Gamma$ is the line-shape operator, the \pm sign in the parentheses is assigned for absorption and PL spectra, respectively. The term $\omega_{00}$ is the electronic origin frequency, and $\omega_{p}$ is the phonon frequency. The HR factor is proportional to the squared displacement of the potential energy minima between the ground $\left(S_{0}\right)$ and

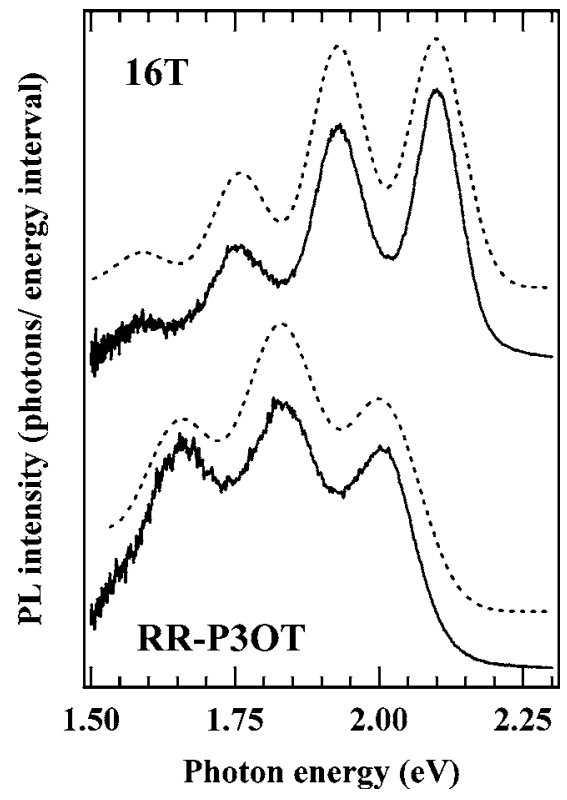

FIG. 8. Examples of the results of the best-fit Franck-Condon analysis (dotted lines) of the PL spectra (solid lines) measured at $4 \mathrm{~K}$ for $16 \mathrm{~T}$ and RR P3OT. For clarity, the dotted lines are offset from the spectral fit.

excited $\left(S_{1}\right)$ states as a function of the configuration coordinate, $\Delta Q_{i}^{2}$. Moreover, the HR factor has a relation with the total relaxation energy $E_{\text {rel }}$,

$$
E_{r e l}=\sum_{i} S_{i} \hbar \omega_{p, i}
$$

and thus gives a measure of the strength of the electronphonon coupling. ${ }^{47}$ Here all active phonon modes coupled to the $S_{0}-S_{1}$ electronic transition of the system should be essentially taken into consideration in the FC analysis of the optical spectra according to Eq. (3). Yet, since the spectra in Figs. 2 and 3 did not show contributions of two, or more, vibronic oscillators that is probably due to inhomogeneous broadening, only a single phonon mode with Gaussian line shape is assumed in the FC analyses.

Concerning the FC analysis of a PL spectrum, the importance of including the term $n_{f}^{3}(\hbar \omega)^{3}$, where $n_{f}$ is the real part of the refractive index at the photon energy $\hbar \omega$, has recently been pointed out as the product of the right-hand side in Eq. (3) where the spectrum is expressed in units of photons/ energy interval. ${ }^{48} \mathrm{We}$ attempted to use the refractive index of PP for $n_{f}{ }^{49}$ but its third power was found to have a negligible $\omega$ dependence in the PL spectral region. We thus simply multiplied the term $(\hbar \omega)^{3}$ in the right-hand side in Eq. (3) during the analytical procedure. The following parameters were obtained from the FC analysis of the PL spectra: the Gaussian full width at half maximum (FWHM), the 0-0 transition energy $\left(E_{0}\right)$, the HR factor $(S)$, and the mean phonon energy $\left(\hbar \omega_{p}\right)$.

The FC analysis was able to account for the observed PL spectra in Figs. 2 and 3. Examples of the spectral fitting using the FC analysis for the $16 \mathrm{~T}$ and RR P3OT samples are shown in Fig. 8. The derived parameters are summarized in 
TABLE III. The parameters determined from the best fittings of Franck-Condon analyses to the steady-state PL spectra at $4 \mathrm{~K}$; the Gaussian full width at half maximum (FWHM), the PL 0-0 transition energy $\left(E_{0}\right)$, the Huang-Rhys factor $(S)$, the average phonon energy $\left(\hbar \omega_{p}\right)$, and the relaxation energy $\left(E_{r e l}=S \hbar \omega_{p}\right)$.

\begin{tabular}{cccccc}
\hline \hline Sample & $8 \mathrm{~T}$ & $12 \mathrm{~T}$ & $16 \mathrm{~T}$ & $\mathrm{RR}$ & $\mathrm{RRa}$ \\
\hline FWHM & 76.2 & 66.2 & 67.4 & 90.5 & 116 \\
$(\mathrm{meV})$ & & & & & \\
$E_{0}(\mathrm{eV})$ & 2.25 & 2.13 & 2.10 & 2.01 & 2.06 \\
$S(-)$ & 1.44 & 1.37 & 1.25 & 1.79 & 1.28 \\
$\hbar \omega_{p}(\mathrm{meV})$ & 171 & 171 & 170 & 177 & 182 \\
$E_{r e l}(\mathrm{meV})$ & 246 & 234 & 213 & 317 & 233 \\
$S / E_{0}\left(\mathrm{eV}^{-1}\right)$ & 0.64 & 0.64 & 0.60 & 0.89 & 0.62 \\
\hline \hline
\end{tabular}

Table III. Comparison of the FWHM values shows that the oligomer samples give narrower linewidths than that of the polymers. This is attributed to the elimination of inhomogeneous broadening due to the chain-length distribution. It is also apparent that the well-defined stereoregularity in substituent positions of RR P3OT reduces inhomogeneity in the PL linewidth, when compared to the poorly stereoregular structure of RRa P3OT.

The mean phonon energy, $\hbar \omega_{p}$, obtained from the FC analyses is $170-171 \mathrm{meV} \quad\left(\sim 1380 \mathrm{~cm}^{-1}\right)$, and $177-182 \mathrm{meV}\left(\sim 1450 \mathrm{~cm}^{-1}\right)$ for the oligomers and polymers, respectively. The observed phonon modes are restricted to the ones coupled to an electronic transition and can be therefore detected by Raman spectroscopy. For oligoand polythiophenes, strong Raman signals around $1450 \mathrm{~cm}^{-1}$ are commonly observed regardless of the chain length. ${ }^{50}$ These Raman vibrational modes are generally assigned primarily to a longitudinal collective vibration involving stretching of the $\mathrm{C}=\mathrm{C}$ bonds and shrinkage of the $\mathrm{C}-\mathrm{C}$ bonds. Therefore, our results indicate that the electronic transition in the polymers is strongly coupled to the common (viz. ca. $1450 \mathrm{~cm}^{-1}$ ) vibrational modes. In the oligomers the electronic transition may also couple with other vibrational modes that have lower frequencies, e.g., the ring $\mathrm{C}-\mathrm{C}$ stretching mode $\left(\sim 1380 \mathrm{~cm}^{-1}\right) .51$

It is clear from the FC analysis that the parameter $S$ ascribed to the oligomers decreases with chain length. Related to this, a linear increase in the HR factor versus $1 / m$ (where $m$ is the number of chain units) was reported previously in PPV oligomers up to the 5 -mer. ${ }^{52}$ A similar plot that demonstrates this quasilinear relation against $1 / m$ is shown in Fig. 4 for our oligothiophene samples. This indicates that the linear relation between $S$ and $1 / \mathrm{m}$ almost holds true for long oligothiophenes at the quasipolymer level as well as for the shorter PPV oligomers. In fact, the HR factor divided by the PL 0-0 peak energy, $S / E_{0}$, is found to give a nearly constant value among the oligomer samples; see Table III. This finding suggests that the electron-phonon coupling in oligomers should become weak as the ECL increases.

The HR factor of RRa P3OT (1.28) is found to be close to that of $16 \mathrm{~T}(1.25)$. According to the relationship between the chain length and the HR factor identified for the oligomer samples, this suggests that the ECLs of RRa P3OT and $16 \mathrm{~T}$ are similar. Indeed, the $S / E_{0}$ values of RRa P3OT and $16 \mathrm{~T}$ are also very close to each other (Table III). On the other hand, the RR P3OT sample exhibits the largest $S$ value despite its expected long chain length and its $S / E_{0}$ value of 0.89 is much larger than those of the other samples $(0.60-$ 0.64). A PL spectrum with a large HR factor was previously reported for RR poly(3-hexylthiophene) in neat film and was interpreted as resulting from signal overlap arising from inter- and intrachain excitons. ${ }^{53}$ In this study, however, such an overlap of signals cannot be expected since interchain interaction is almost eliminated by means of extreme dilution with PP. The reason why RR P3OT in PP has a large HR factor is not fully understood at present, but one of the possible causes could be the stereoregular head-to-tail structure of RR P3OT. Namely, the head-to-tail structure of RR P3OT is presumed to have the advantage of being able to exhibit strong electron-phonon coupling. The results of our work with the oligomers have allowed us to link increasing electron-phonon coupling to a reduction of the ECL or to an enhancement of the stereoregularity in the molecular structure. Thus, the primary factor that reduces electron-phonon coupling should be a kind of geometric distortion that increases with chain length.

In contrast to the case observed in the PL spectra, the absorption spectra of the oligomers in Fig. 3 were not wellfitted with the FC analysis, even though their PLE spectra were adopted instead of the absorption spectra. However, the comparison between the first and second vibronic peaks in the PLE spectra of the oligomer samples in Fig. 3 suggests a decrease in the HR factor with increasing chain length; this is similar to the trend observed in the PL spectra. Therefore it is concluded that even in the absorption transition the strength of electron-phonon coupling is reduced as a function of increasing chain length.

\section{B. Intrachain exciton migration}

Figures 6 and 7 demonstrated that RRa P3OT and $16 \mathrm{~T}$ diluted in PP exhibit a transient PL redshift whereas RR $\mathrm{P} 3 \mathrm{OT}$ does not. This suggests that the transient redshift in our samples takes place due to poor stereoregularity in the substituent groups (they are highly stereoregular in RR $\mathrm{P} 3 \mathrm{OT})$. If poor stereoregularity enables an energy gradient of excitons to form within a single chain, the transient redshift may originate from intrachain migration. A transient redshift could also take place in the course of a vibrational relaxation or of structural deformation following photoexcitation. Indeed, a model assuming the presence of the PL from upper vibrational levels was recently proposed to explain a transient redshift in a neat PT-derivative film. ${ }^{33}$ The vibrational relaxation and structural deformation, if any, are expected to take place independently of the size of the molecule or the substituent position. However, our range of samples gave different magnitudes for the transient shifts. As a result, we conclude that these transient shifts originate from intrachain exciton migration. Yet the presence of the transient PL shift due to structural deformation cannot be entirely ruled out and is discussed in the following section.

The observations of the intrachain exciton migration enable us to derive several significant physical properties of 
conjugated polymers and oligomers. The exciton migration towards lower energy sites occurs, once the electronic energy gradient is generated between species with different ECLs. Therefore, the observation of intrachain migration demonstrates the presence of an energy gradient within a molecule. The results of $16 \mathrm{~T}$, as well as of RRa P3OT, suggest that such an intrachain energy gradient exists there. It is hence expected that two or more exciton states with different ECLs may exist even in $16 \mathrm{~T}$.

The phenomenon of intrachain migration is clearly seen for RRa P3OT diluted in PP (Fig. 6). From its time-resolved spectra the presence of a component with a larger redshift $(\sim 30 \mathrm{meV})$ compared to the moment shift $(\sim 12 \mathrm{meV}$, see Fig. 7) can be inferred. Considering the results of the steadystate measurements (see Fig. 2) described in Sec. III A, the observed migration shift in Fig. 6 renders a picture of the exciton energy transfer from species with shorter ECLs (giving primary absorption signals) to the species with longer ECLs (giving primary PL signals). Furthermore, in Fig. 6, the latter species are found to give a constant PL line shape with time below the peak $(2.07 \mathrm{eV})$. This finding indicates that the PL signals for species with longer ECLs are caused not only by a component transferred from the species with shorter ECLs but also by a component that rapidly migrates and/or has no transient shift. These mechanisms facilitate to reduce the moment estimation and result in the underestimation of the real migration shift $(\sim 30 \mathrm{meV})$.

Finally, this intrachain migration was observed in the time scale of several $100 \mathrm{ps}$, which is comparable to that of interchain migration reported for neat films of conjugated polymers. ${ }^{28-33}$ This is surprising because we usually expect an exciton transfer to be much faster in intrachain processes than that in interchain ones. Although rapid intrachain process beyond the time resolution of the present setup may exist, our results suggest that severe distortion which hinders the intrachain transfer is present in a chain of usual conjugated polymers, unless the substituents themselves are coupled to each other in a head-to-tail manner.

\section{Mechanism of PL for oligo- and polythiophenes diluted in solid matrix}

In this section we discuss the electronic transition in a system with strong electron-phonon coupling using the notion of configuration coordinate assuming a harmonic oscillator for vibrational modes; see Fig. 9. This allows us to summarize the intrachain PL dynamics for the oligo- and the polythiophene derivatives. Along the vertical absorption transition in Fig. 9, the equilibrium position of the vibrational mode is displaced depending on the strength of the coupling between the generated excitons and phonons. The displacement of the equilibrium position, $\Delta Q_{\mathrm{ab}}$, is directly related to the HR factor of the absorption spectra.

Following the absorption transition, two major processes should be involved before leading to the PL transition: (1) exciton migration (see the previous section) and (2) a change in the ring torsional angle around the molecular axis induced by photoexcitation. The importance of the latter process has been theoretically developed for explaining the optical spec-

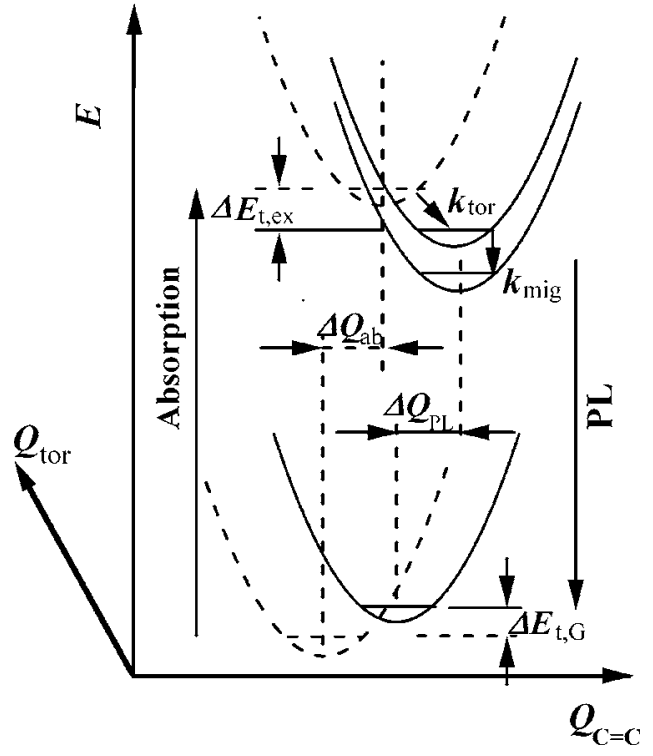

FIG. 9. A schematic description of the energy diagram illustrating the absorption and PL transitions. The notion of the configuration coordinate with a harmonic-oscillator type vibrational mode is assumed in the layout of this diagram. The horizontal axis shows a displacement of the equilibrium separation of the primary $\mathrm{C}=\mathrm{C}$ stretching vibrational mode. The axis from the front to the back of the figure represents the degree of ring-torsion motion. The potential energy curves are drawn with the broken and the straight lines, respectively, for the states before and after the change in the ringtorsion angle. Intrachain migration is assumed to be present. $k_{\mathrm{tor}}$ and $k_{\text {mig }}$ represent the processes of the molecular distortion (torsional change) and intrachain exciton migration, respectively. $\Delta Q_{\mathrm{ab}}$ and $\Delta Q_{\mathrm{PL}}$ represent the displacements of the configuration on the absorption and PL transitions, respectively. $\Delta E_{\mathrm{t}, \text { ex }}$ and $\Delta E_{\mathrm{t}, \mathrm{G}}$ represent the energy changes induced by the molecular distortion in the excited and ground states, respectively.

tra of short conjugated oligomers. ${ }^{46,54-56}$ The basic idea originates from the experimental observation that $p$-phenylene oligomers have significant Stokes-shifted absorption and PL spectra, whereas their ladder-type analogs exhibit spectral patterns with minor Stokes shifts owing to the presence of saturated bridges between each ring. ${ }^{57,58} \mathrm{Al}$ though its theoretical interpretation is somewhat controversial it would be true that some Stokes shift is caused by the difference in torsional angle between the molecular configuration at the absorption and PL transitions. In short, oligomers have a relatively coplanar configuration at the PL transition leading to a lower energy transition. Since the change in torsional angle is a kind of vibronic relaxation, it can be illustrated using configuration coordinates; it is the transfer between the front and back surfaces of Fig. 9. This model is also applicable to thiophene derivatives because the bonds between thiophene rings are expected to have no major hindrance during torsional motions. Moreover, according to the Franck-Condon principle, following the PL transition (cf. coplanar configuration) a recover process of the change in torsional angle is required in the ground state.

In this sense, the torsional change, its recovery process, and the intrachain exciton migration described in the previous section are possible origins to cause a Stokes shift in our 
samples. As shown in Figs. 2 and 3, all of our samples are found to exhibit a Stokes shift $(\sim 60-70 \mathrm{meV})$, the values of which are listed in Table I. In the previous section, the intrachain migration was interpreted to take place between species with different ECL, whereas the Stokes shift in Table I was determined based on the PL and PLE peaks of the species with longer ECLs. Therefore the Stokes shift here is concluded to originate not from the intrachain migration but from the molecular distortion and its recovery process. This conclusion is consistent with the data presented in Table I demonstrating that the Stokes shift values are similar to each other $(60-70 \mathrm{meV})$. This is because the extent of molecular torsion is expected to be nearly equal among the samples with different chain lengths. We therefore suggest that the constant Stokes shift due to the molecular distortion would be a common spectral feature in thiophene derivatives that has no dependency on chain length. In Fig. 4, the linear fits of the $0-0$ peaks against the reciprocal of ring number indicated that the coplanarity of the thiophene rings should be almost preserved in absorption and PL transitions. It was at the same time suggested that a variation in the resonance integral between neighboring thiophene rings should be minor. Therefore, we conclude that although the molecular distortion at the transitions causes a change in the transition energy the extent should not be so large as to reduce the magnitude of the resonance integral.

No transient spectral shifts corresponding to a 60-70 meV Stokes shift were observed in the time-resolved PL spectra in Fig. 6. This suggests that the molecular distortion should occur within the limit of time resolution $(\sim 50 \mathrm{ps})$ following photoexcitation. This finding is consistent with the recent report on the transient absorption measurements for the 13-mer oligothiophene in solution that suggested that the dynamic redshift occurs within 2 ps. ${ }^{59}$ Furthermore, it is interesting to note that the magnitude of the dynamic redshift was estimated to be $24.2 \mathrm{meV} .{ }^{59}$ The value of the dynamic shift is close to the half of the Stokes shift $(30-35 \mathrm{meV})$ determined in this present study, which almost corresponds to the energy shift at the torsion change in the excited state, $\Delta E_{\mathrm{t} \text { ex }}$ in Fig. 9.

Based on the above considerations, the optical spectra of thiophene derivatives are expected to give at least a Stokes shift of $60-70 \mathrm{meV}$. If the observed Stokes shift is more than $70 \mathrm{meV}$ then its surplus should be attributed to the migration shift. For instance, the large Stokes shift between the absorption and PL spectra of the RRa P3OT in Fig. 2 $(>0.4 \mathrm{eV})$ suggests that the majority of the species with shorter ECLs migrate more than $0.3 \mathrm{eV}$ towards the species with longer ECLs. As long as the PL signals in the neat film samples originate finally from intrachain excitons, this idea should also be applicable to interchain migration. Moreover the intrachain PL process for other conjugated polymers can also be considered based on the mechanism proposed in this study, assuming that the magnitude of the Stokes shift due to the molecular distortion processes can be properly determined.

\section{CONCLUSIONS}

The PL spectra for long-chained oligothiophenes, RR and RRa P3OTs diluted in the inactive solid matrix polypropyl- ene, were measured and analyzed in order to unveil the intrachain PL processes in conjugated polymers. The analyses of the spectra presented several significant findings, which are summarized below.

The oligothiophenes gave resolved 0-0 peaks in both of their absorption and PL spectra. These $0-0$ peaks were confirmed to show a good linear relationship when plotted against the reciprocal of the ring number. A small difference in the value of the slope was found between the absorption and PL transitions. This difference was ascribed to a minor variation in the planarity of thiophene rings between the ground state and the excited state relevant to the PL transition.

Franck-Condon analyses were carried out for the PL spectra. The strength of the electron-phonon coupling represented by the HR factor was demonstrated to become weak with increasing chain length. In contrast, RR P3OT exhibited the largest HR factor and hence showed the presence of strong electron-phonon coupling in RR. We conclude that some sort of geometric distortion occurs, which reduces the strength of electron-phonon coupling and this effect is proportional to the extension of the effective conjugation length. We also conclude that the coupling strength largely depends on stereoregularity in the chain conformation.

Transient PL measurements measured at $4 \mathrm{~K}$ showed that the PL decay rate is somewhat slower in the oligomers than in the polymers. This is attributed to the feature in the nonradiative decay process that is enhanced with increased chain length. Possible mechanisms to explain this phenomenon were proposed; ISC paths to other than the nearest neighbor $T_{n}$ state are generated with increasing the chain length, and/or the probability of the excitons to be quenched by extrinsic defects increases with the chain length. In addition, even in the 16-mer oligothiophene the time-resolved PL spectrum showed the presence of intrachain exciton migration whereas RR P3OT did not exhibit these. These features indicate that intrachain migration depends on the degree of stereoregularity of the chain conformation.

Based on the results of continuous wave and transient PL experiments, we conclude that a change in the torsion angle around the thiophene ring-to-ring bonds occurs following the absorption transition in oligo- and polythiophenes regardless of the chain length. We also conclude that the torsional change and its recovery process give rise to a constant Stokes shift $(60-70 \mathrm{meV})$. If the redshift is more than $70 \mathrm{meV}$ then its surplus should be assigned to the contribution of the exciton migration effect, including interchain migration events.

\section{ACKNOWLEDGMENTS}

H. H. thanks the Japanese Ministry of Education, Culture, Sports, Science \& Technology (Grants No. 17204026 and No. 17654083) and SICP/JST. Y. A. thanks the Japanese Ministry of Education, Culture, Sports, Science \& Technology for a Grant-in-Aid for Scientific Research (Category B, No. 16350022, and Priority Areas, No. 16038217). We are pleased to thank Andrew Gall of the University of Glasgow for the correction of English text of this manuscript. 
*Corresponding author. Email address: kkane@sci.osaka-cu.ac.jp

${ }^{1}$ J. H. Burroughes, D. D. C. Bradley, A. R. Brown, R. N. Marks, K. Mackay, R. H. Friend, P. L. Burns, and A. B. Holmes, Nature 347, 539 (1990).

${ }^{2}$ G. Yu, J. Gao, J. C. Hummelen, F. Wudl, and A. J. Heeger, Science 270, 1789 (1995).

${ }^{3}$ H. Sirringhaus, N. Tessler, and R. H. Friend, Science 280, 1741 (1998).

${ }^{4}$ Relaxation of Polymers, edited by T. Kobayashi (World Scientific, Singapore, 1993).

${ }^{5}$ Z. V. Vardeny and X. Wei, in Handbook of Conducting Polymers II, edited by T. A. Skotheim, R. L. Elsenbaumer, and J. R. Reynolds (Marcel Dekker, New York, 1997), p. 639.

${ }^{6}$ E. M. Conwell, in Organic Electronic Materials, edited by R. Farchioni and G. Grosso (Springer, Berlin, 2001), p. 127.

${ }^{7}$ T. A. Chen, X. M. Wu, and R. D. Rieke, J. Am. Chem. Soc. 117, 233 (1995).

${ }^{8}$ G. Rumbles, I. D. W. Samuel, L. Magnani, K. A. Murray, A. J. DeMello, B. Crystall, S. C. Moratti, B. M. Stone, A. B. Holmes, and R. H. Friend, Synth. Met. 76, 47 (1996).

${ }^{9}$ L. Magnani, G. Rumbles, I. D. W. Samuel, K. A. Murray, S. C. Moratti, A. B. Holmes, and R. H. Friend, Synth. Met. 84, 899 (1997).

${ }^{10}$ M. Theander, M. Svensson, A. Ruseckas, D. Zigmantas, V. Sundström, M. R. Andersson, and O. Inganäs, Chem. Phys. Lett. 337, 277 (2001).

${ }^{11}$ S. Luzzati, P. Elmino, and A. Bolognesi, Synth. Met. 76, 23 (1996).

${ }^{12}$ M. R. Andersson, M. Berggren, T. Olinga, T. Hjertberg, O. Inganäs, and O. Wennerström, Synth. Met. 85, 1383 (1997).

${ }^{13}$ R. Österbacka, C. P. Au, X. M. Jiang, and Z. V. Vardeny, Science 287, 839 (2000).

${ }^{14}$ M. Fakis, I. Polyzos, G. Tsigaridas, V. Giannetas, P. Persephonis, I. Spiliopoulos, and J. Mikroyannidis, Phys. Rev. B 65, 195203 (2002).

${ }^{15}$ T. Virgili, D. Marinotto, C. Manzoni, G. Cerullo, and G. Lanzani, Phys. Rev. Lett. 94, 117402 (2005).

${ }^{16}$ K. Kanemoto, M. Shishido, T. Sudo, I. Akai, H. Hashimoto, and T. Karasawa, Chem. Phys. Lett. 402, 549 (2005).

${ }^{17}$ K. Kanemoto, M. Shishido, T. Sudo, I. Akai, T. Karasawa, and Y. Agari, Synth. Met. 155, 162 (2005).

${ }^{18}$ H. Sirringhaus, P. J. Brown, R. H. Friend, M. M. Nielsen, K. Bechgaard, B. M. W. Langeveld-Voss, A. J. H. Spiering, R. A. J. Janssen, E. W. Meijer, P. Herwig, and D. M. de Leeuw, Nature 401, 685 (1999).

${ }^{19}$ Handbook of Oligo- and Polythiophenes, edited by D. Fichou (Wiley-VCH, Weinheim, 1999).

${ }^{20}$ F. Charra, D. Fichou, J. M. Nunzi, and N. Pfeffer, Chem. Phys. Lett. 192, 566 (1992).

${ }^{21}$ H. Chosrovian, D. Grebner, and S. Rentsh, Synth. Met. 52, 213 (1992).

${ }^{22}$ Y. Kanemitsu, N. Shimizu, K. Suzuki, Y. Shiraishi, and M. Kuroda, Phys. Rev. B 54, 2198 (1996)

${ }^{23}$ R. S. Becker, J. S. deMelo, A. L. Macanita, and F. Elisei, J. Phys. Chem. 100, 18683 (1996).

${ }^{24}$ J. Gierschner, H. G. Mack, H. J. Egelhaaf, S. Schweizer, B. Doser, and D. Oelkrug, Synth. Met. 138, 311 (2003).

${ }^{25}$ H. Nakanishi, N. Sumi, Y. Aso, and T. Otsubo, J. Org. Chem. 63, 8632 (1998).

${ }^{26}$ N. Sumi, H. Nakanishi, S. Ueno, K. Takimiya, Y. Aso, and T.
Otsubo, Bull. Chem. Soc. Jpn. 74, 979 (2001).

${ }^{27}$ T. Izumi, S. Kobashi, K. Takimiya, Y. Aso, and T. Otsubo, J. Am. Chem. Soc. 125, 5286 (2003).

${ }^{28}$ G. R. Hayes, I. D. W. Samuel, and R. T. Phillips, Phys. Rev. B 52, R11569 (1995).

${ }^{29}$ G. R. Hayes, I. D. W. Samuel, and R. T. Phillips, Phys. Rev. B 54, R8301 (1996).

${ }^{30}$ L. M. Herz and R. T. Phillips, Phys. Rev. B 61, 13691 (2000).

${ }^{31}$ L. M. Herz, C. Silva, R. T. Phillips, S. Setayesh, and K. Müllen, Chem. Phys. Lett. 347, 318 (2001).

${ }^{32}$ T. Kobayashi, J. Hamazaki, M. Arakawa, H. Kunugita, K. Ema, K. Ochiai, M. Rikukawa, and K. Sanui, Phys. Rev. B 62, 8580 (2000).

${ }^{33}$ T. Kobayashi, J. I. Hamazaki, H. Kunugita, K. Ema, T. Endo, M. Rikukawa, and K. Sanui, Phys. Rev. B 67, 205214 (2003).

${ }^{34}$ T. Yamashiro, Y. Aso, T. Otsubo, H. Tang, Y. Harima, and K. Yamashita, Chem. Lett. 443 (1999).

${ }^{35}$ G. Bidan, A. De Nicola, V. Enée, and S. Guillerez, Chem. Mater. 10, 1052 (1998).

${ }^{36}$ M. Theander, O. Inganäs, W. Mammo, T. Olinga, M. Svensson, and M. R. Andersson, J. Phys. Chem. B 103, 7771 (1999).

${ }^{37}$ K. Kanemoto, I. Akai, H. Hashimoto, T. Karasawa, Y. Aso, and T. Otsubo (unpublished).

${ }^{38}$ L. Lemmer, R. F. Mahrt, Y. Wada, A. Greiner, H. Bässler, and E. O. Göbel, Appl. Phys. Lett. 62, 2827 (1993).

${ }^{39}$ H. D. Burrows, J. S. de Melo, C. Serpa, L. G. Arnaut, M. De G. Miguel, A. P. Monkman, I. Hamblett, and S. Navaratnam, Chem. Phys. 285, 3 (2002).

${ }^{40}$ D. Beljonne, J. Cornil, R. H. Friend, R. A. J. Janssen, and J. L. Brédas, J. Am. Chem. Soc. 118, 6453 (1996).

${ }^{41}$ B. Kraabel, D. Moses, and A. J. Heeger, J. Chem. Phys. 103, 5102 (1995).

${ }^{42}$ C. M. Heller, I. H. Campbell, B. K. Laurich, D. L. Smith, D. D. C. Bradley, P. L. Burn, J. P. Ferraris, and K. Müllen, Phys. Rev. B 54, 5516 (1996).

${ }^{43}$ W. P. Su, J. R. Schrieffer, and A. J. Heeger, Phys. Rev. B 22, 2099 (1980).

${ }^{44}$ A. J. Heeger, S. Kivelson, J. R. Schrieffer, and W. P. Su, Rev. Mod. Phys. 60, 781 (1988).

${ }^{45}$ M. Pope and C. E. Swenberg, Electronic Processes in Organic Crystals (Clarendon, Oxford, 1982).

${ }^{46}$ J. Gierschner, H. G. Mack, L. Lüer, and D. Oelkrug, J. Chem. Phys. 116, 8596 (2002).

${ }^{47}$ D. Dragoman and M. Dragoman, Optical Characterization of Solids (Springer, Berlin, 2002).

${ }^{48}$ P. K. H. Ho, J. S. Kim, N. Tessler, and R. H. Friend, J. Chem. Phys. 115, 2709 (2001).

${ }^{49}$ H. Fujimatsu, Y. Ideta, H. Nakamura, H. Usami, and S. Ogasawara, Polym. J. (Tokyo, Jpn.) 33, 543 (2001).

${ }^{50}$ M. D. Zoppo, C. Castiglioni, P. Zuliani, and G. Zerbi, in Handbook of Conducting Polymers II, edited by T. A. Skotheim, R. L. Elsenbaumer, and J. R. Reynolds (Marcel Dekker, New York, 1997), p. 765.

${ }^{51}$ G. Louarn, M. Trznadel, J. P. Buisson, J. Laska, A. Pron, M. Lapkowski, and S. Lefrant, J. Phys. Chem. 100, 12532 (1996).

${ }^{52}$ J. Cornil, D. Beljonne, C. M. Heller, I. H. Campbell, B. K. Laurich, D. L. Smith, D. D. C. Bradley, K. Müllen, and J. L. Brédas, Chem. Phys. Lett. 278, 139 (1997).

${ }^{53}$ P. J. Brown, D. S. Thomas, A. Köhler, J. S. Wilson, J. S. Kim, C. M. Ramsdale, H. Sirringhaus, and R. H. Friend, Phys. Rev. B 
67, 064203 (2003).

${ }^{54}$ F. Momicchioli, M. C. Bruni, and I. Baraldi, J. Phys. Chem. 76, 3983 (1972).

${ }^{55}$ S. Karabunarliev, E. R. Bittner, and M. Baumgarten, J. Chem. Phys. 114, 5863 (2001).

${ }^{56}$ G. Heimel, M. Daghofer, J. Gierschner, E. J. W. List, A. C. Grimsdale, K. Müllen, D. Beljonne, J. L. Brédas, and E. Zojer, J.
Chem. Phys. 122, 054501 (2005).

${ }^{57}$ J. Grimme and U. Scherf, Macromol. Chem. Phys. 197, 2297 (1996).

${ }^{58}$ T. Pauck, H. Bässler, J. Grimme, U. Scherf, and K. Müllen, Chem. Phys. 210, 219 (1996).

${ }^{59}$ A. Ozawa, K. Takimiya, T. Otsubo, and T. Kobayashi, Chem. Phys. Lett. 409, 224 (2005). 